\title{
Hybrid WLAN for Data Dissemination Applications*
}

\author{
Ching-Ju Lin and Cheng-Fu Chou \\ Department of Computer Science and Information Engineering, \\ National Taiwan University, Taipei, Taiwan, ROC \\ \{shauk, ccf\}@cmlab.csie.ntu.edu.tw
}

\begin{abstract}
In traditional infrastructure-based IEEE 802.11 wireless LAN network, all the mobile nodes associated themselves with an access point (AP) share the same wireless channel with other mobile devices. In such wireless network environment, there are two major inefficiencies: (a) load imbalance among different APs: when the distribution of the location of mobile nodes is non-uniform, this might result in a situation where a lot of mobile nodes access a single AP and the rest of APs are idle, and (b) poor resource utilization: most of the mobile nodes contend for the wireless channels of APs while there are some unused and available wireless channels, which can be used to set up ad hoc groups. In this paper, we propose the hybrid WLAN (H-WLAN) framework to address these two problems. First, our H-WLAN framework provides the adaptive service selection module for the mobile node to figure out a better wireless channel and the server to get its service. Second, the popularity-aware channel management module is able to efficiently integrate the ad hoc mode with the infrastructure mode by allowing mobile nodes to set up a new ad hoc group and then share their resources with other nodes within that group. The simulation results show that our $H$-WLAN framework can substantially (a) achieve high utilization and load balance among wireless channels with lower control overhead, and (b) provide better quality of service for the mobile nodes.
\end{abstract}

\section{Introduction}

Wireless local area networks have come into great use in recent years. Although a variety of wireless network technologies have been in the market, wireless LANs based on the IEEE 802.11 standard are the most popular. There are two operation modes, i.e., the infrastructure mode and the ad hoc mode, specified in IEEE 802.11 standard. In the ad

* This work was partially supported by the National Science Council and the Ministry of Education of ROC under the contract No. NSC922622-E-002-002 and 89E-FA06-2-4-8. hoc mode, all mobile nodes are free to directly communicate with each other in a peer-to-peer manner. Another option of the operation mode in WLAN is the infrastructure mode, where each mobile node associates itself with an access point (AP) within its direct transmission range. The AP is connected to a wired network and provides a conduit to the external network for the mobile nodes. Communications can only take place between the wireless node and the AP but not directly between the wireless nodes.

In this paper, we consider the problem of distributing popular data at the server to users in a wireless LAN environment. The data dissemination problems correspond to a set of important applications. These applications include distribution of course slides in a class, dissemination of useful information in the info-station, online documents distribution in a conference, and many more. In a traditional infrastructure-based WLAN, all communications will go through the APs, but an AP can only provide a certain amount of bandwidth (e.g., 802.11b is $11 \mathrm{Mbps}$ ) for all users within its transmission coverage. In such wireless network environment, there are two major inefficiencies: (a) load imbalance problem among the APs: when the distribution of the location of the mobile nodes is non-uniform, this might lead to a situation where a lot of nodes access a single AP but other APs are idle, and (b) poor resource utilization: most of the nodes contend for the wireless channel of the AP while there are still some unused but available wireless channels, which can be used to set up ad hoc groups. When the number of users increases within an AP's cell, the contention for the wireless channel will lead to more collisions, degradation in system throughput, and longer data delivery time for the mobile nodes. To increase the system throughput and reduce the user's response time, a simple solution is to allocate more bandwidth for users by installing more APs, which use different wireless channels, but this approach is expensive and not flexible to deal with the "temporary" fluctuation of the network load.

In a wireless network environment, an important observation for the data dissemination applications is that most local users are interested in nearly the same set of objects. 
In other words, since the requested objects of local users are similar, the mobile nodes can form a local ad hoc group to communicate with each other for sharing those objects without going through the APs until they have to switch back to the infrastructure mode. Furthermore, setting up the local ad hoc groups, which use different channels from the APs, can mitigate the contention and collisions in the infrastructure-based APs channels. Therefore, if we can efficiently integrate the ad hoc mode and the infrastructure mode, such hybrid WLAN is able to increase both the system and user's throughput, and provides a better and consistent service.

However, there are several important issues that need to be addressed in order to to design a high-performance hybrid WLAN framework. The first one is the service selection problem between different ad hoc groups and infrastructure-based networks. The second one is channel allocation problem including when to set up a new ad hoc group and which channel should be assigned to that group. An inappropriate service selection or channel allocation mechanism may lead to congestion in some channels or servers while others might be idle. In our H-WLAN framework, we use the adaptive service selection module for the mobile node to figure out a better channel-server selection to get its service, while achieving higher throughput for the mobile node and the load balance between the wireless channels. The popularity-aware channel management module is used to determine the proper wireless channel allocation to fast and correctly react the actual network condition. The simulation results show that our H-WLAN can result in significant performance improvements. That is, the H-WLAN can efficiently integrate the ad hoc and infrastructure modes and achieve load balance among wireless channels, better channel utilization, and higher throughput for both the system and the mobile nodes.

The remainder of the paper is organized as follows. In section 2, we review the related works on integrating the ad hoc wireless network with infrastructure-based WLAN. The H-WLAN framework is described in Section 3. Section 4 presents a performance study of our proposed framework. At last, the conclusion is given in Section 5.

\section{Related Work}

In general, most of works on ad hoc networks focus on providing routing technologies to improve the efficiency of content distribution applications. In [9] the authors propose two schemes, i.e., CacheData and CachePath, that the mobile nodes cache the popular data or data path for others in the same ad hoc network whereas our H-WLAN system aims to improve data access in the infrastructurebased WLAN environment with the help of multiple ad hoc groups.
On the other hand, some works based on infrastructure mode network aim to improve the performance of content dissemination and provide the QoS. In WICAT system [7], each mobile node has a list of interested objects, and these objects may be located at different infostations. Only when a mobile node moves close to an infostation, it is able to download those objects that are available at that infostation and match user's preference. In [8], their framework supports a map-on-the-go application, i.e., users move between infostations, and each infostation provides relevant maps to these users. These two systems focus on designing an efficient infrastructure-based wireless system to distribute useful objects and information for users; however, our framework is a hybrid wireless system integrating the ad hoc mode with infrastructure modes.

Other frameworks or systems, include [6], [1], [10], NUMI [5], and $M^{2}$-WLAN [2], use peer-to-peer communication to assist the infrastructure-based system for data exchange or distribution. [10] did an initial study on using non-cooperative mechanism, e.g., peer-to-peer communication, to improve the efficiency of content distribution for the infostation system. Two encountered mobile nodes check the file content lists of each other, identify some files they need, and exchange those files. In NUMI [5], the APs collaborate among themselves and can predict the mobile node's location in the near future. Using this information, the AP is able to select another mobile node, which is likely to move around the vicinity of the destination mobile node, to piggyback the requested data for the destination mobile node. However, how to predict the moving behavior of mobile nodes has the great effect on the performance of the NUMI system. In the $M^{2}$-WLAN framework [2], besides communicating with the APs, the mobile node can also switch to ad hoc mode to connect with another node under the administration of the AP, the central manager that assigns channels and keeps the information for all mobile nodes. Both NUMI and $M^{2}$-WLAN frameworks are for host-centric applications while our H-WLAN framework is for data dissemination applications. In addition, the peer-to-peer communication is a single-hop connection in NUMI and $M^{2}$-WLAN systems but can be a multi-hop connection in our H-WLAN framework, i.e., the transmission coverage of our framework can be extended by relaying data through multiple nodes.

Lastly, much work (as described in [3]) has been done in addressing server selection problems for services replicated across wide-area networks. We note that our work differs from them in (i) our adaptive service selection module can maintain a better tradeoff between the load balancing nodes across the service and locating a "closer" service to a node, and (ii) how to manage channel allocation in the H-WLAN framework has a great impact on the performance of the service selection scheme. 


\section{Hybrid WLAN Framework}

In this section, we first give an introduction about how a mobile node can switch its connection between the infrastructure mode and ad hoc mode in the IEEE 802.11 standard and then present our H-WLAN framework.

In a MAC frame header, ToDS and FromDS bits represent the frame to be transmitted into a Distribution System (DS) or from a DS and indicate the meaning of the four address fields in the MAC frame. If both ToDS and FromDS bits are zero, this packet is exchanged between two wireless nodes without an AP involved. If a mobile node runs in the infrastructure-mode, the ToDS bit is set to one and FronDS is set to zero. This means that the mobile node sends a packet to another node via the AP. In this work we assume that a mobile node can change some parameters setting to decide which mode (or wireless channel) it would like to use. That is, a mobile node can switch between the infrastructure mode and ad hoc mode dynamically. Usually there are multiple wireless channels available in a wireless network. For instance, there are 14 channels defined in the DSSS PHY frequency channel plan in the 802.11 standard. Our goal is to make use of the available but unused wireless channels to form some ad hoc groups for assisting the data distribution to relieve the traffic load at the APs and to improve the performance for both the system and the users.

The main challenge of H-WLAN framework is how to efficiently integrate ad hoc mode into the traditional infrastructure-based WLAN environment. To address this challenge in the H-WLAN framework, we propose two major components (a) the adaptive service selection module, and (b) popularity-aware channel management module. After a mobile node joins the wireless network and wants to get the objects, it first uses the service selection module to decide it can get the requested objects from which server through which channel. In popularity-aware channel management module, the H-WLAN system is able to decide when and how to set up a new ad hoc group and to allocate which channel to this group. If a mobile node would like to share its objects with other nodes, the H-WLAN system determines if it is necessary to set up a new ad hoc group or just notifies this node to join the existing ad hoc group to share its resource using the same wireless channel. Next, we discuss each of them in detail accordingly.

\subsection{Adaptive Service Selection Module}

To choose a proper server, the service selection module consists of two phases: one is the resource discovery phase and the other is the server-channel selection phase. The resource discovery phase is that the system first provides the potential server list, i.e., a list of all accessible servers which can provide the requested object, to the re-

\begin{tabular}{||l|l|l||}
\hline object id & channel id & available server id \\
\hline 3 & 1 & 9,8 \\
\cline { 2 - 3 } & 2 & 7 \\
\hline 7 & 1 & 9 \\
\hline
\end{tabular}

Table 1. Group Information Table.

\begin{tabular}{||l|l|l||}
\hline channel id & group leader & status \\
\hline 1 & 9 & on \\
\hline 2 & 7 & on \\
\hline 3 & null & off \\
\hline
\end{tabular}

Table 2. Channel Status Table.

quest mobile node. After the mobile node acquires the potential server list, it can use that information and choose a proper server. For instance, when the mobile node in the infrastructure mode wants to get an object via the wireless network, it might contact with one of the APs within its direct transmission range and then obtain the potential server list from that AP. In order to provide such information, we use the APs as an information-managing center, and each AP maintains a group-information table, which contains three fields: the object id, the wireless channel and the available servers. Each tuple in the server list of object $i$, as shown in Table 1, contains two fields: the first field is the wireless channel used by these corresponding servers in the available server field, and the second field is the available servers, i.e., the nodes hold object $i$ at that moment and in this ad hoc group. The discussions of the maintenance issues, such as how to set up, delete and update the group-information table, are presented in the following subsections.

3.1.1. Resource Discovery Phase: Next, we describe how a mobile node, according to its location, obtains the potential server list below:

1) Within the AP coverage: when the mobile node is within some APs' transmission range, there are two possible cases as follows. If this mobile node is in some ad hoc group, it first needs to switch back to the infrastructure mode without affecting ongoing communication. That is, the mobile node examines if it is involved in the process of any ongoing communications. If so, the mobile node has to wait for the end of those communications and then switch back to infrastructure mode. Otherwise, the mobile node is able to switch back to the infrastructure mode immediately. After the mobile node switches back to the infrastructure mode, the following procedures are the same as those in the infrastructure mode case. If this mobile node is in the infrastructure mode, it sends out the request message to one of those APs and asks for the potential server list. 
2) Beyond the AP coverage: when the mobile node is beyond the transmission range of any AP, it randomly joins an ad hoc group within its direct transmission range and then sends out the request message to the leader of that ad hoc group. After receiving the request message, the group leader processes that message, generates the corresponding request message and switches to the infrastructure mode to send that request message to the AP for that request mobile node. After getting the source list, the group leader forwards the resource information back to the mobile node. Since the group leader is the relay node for those mobile nodes which cannot access any AP, one sufficient condition for the group leader is that it should locate within transmission range of some APs.

As the AP receives the request, it cannot tell if this request is from the intermediate node such as the group leader or not. The AP retrieves the potential server list from the group information table and returns that information to the client. The potential server list records all members that hold the requested objects in each different ad hoc group. In the traditional infrastructure-based 802.11 WLAN, if a mobile node is out of any APs' transmission range, it is unable to set up any wireless connection even if there are some mobile nodes within its transmission range. The intuition behind our work is the mobile node still has the chance to get its requested objects via its neighbor nodes in an ad hoc group even though it cannot connect to any AP directly. Therefore, the data delivery range in H-WLAN is extended via combining the ad hoc mode, and it also lets more users be able to get their requested objects.

3.1.2. Server-Channel Selection Phase: After a mobile node gets the potential server list from the AP, it is likely that there is more than one server in the list. In this subsection, we discuss how the mobile node to choose the suitable server to get its requested objects. Our goal is to maintain the tradeoff between the load balance and the proximity among all available wireless channels so that the system utilization and users' QoS can be improved. Five various methods are presented to select a proper channel-server pair, and the motivations and characteristics of each method as follows:

1) Signal-Strength Method (wlan): In this method, the mobile node connects to the AP with the best signal strength. This method is simple and widely used in current infrastructure-based WLAN. However, as the number of mobile nodes increases and the distribution of the mobile node's location is non-uniform, some APs might have heavy traffic load but the others might be still idle, i.e., it leads to the load imbalance problem. We include this signal-strength method and use it as a baseline approach to compare with.

2) Log-Based Method (wlan-nc): The log-based method is that each AP keeps track of the total number of current flows and includes this information in its broadcasting beacon message. A mobile node might receive several beacon messages from different APs, and can connect to the lightloaded server in order to avoid the congestion. The motivation for this method is to explore the benefit of such simple bookkeeping method.

3) Random-based Method (random): When there is more than one server in the potential server list, the mobile node randomly chooses a server from it and then checks if this server is reachable. If not, the mobile node continues to select another one until it can establish a connection with the chose server. The random-based method is straightforward and able to achieve load balance among the servers. However, the selected server may be far from the request mobile node, or the channel that they use may happen to be congested. We illustrate this effect in the performance evaluation section.

4) Ping-based Methods (ping, pr2, and r2p): To investigate more dynamic and adaptive source selection schemes, we consider the ping-based methods. The ping-based method is that the mobile node pings every source candidates in the potential list and then selects one server after receiving their response messages. The idea of such scheme is to use the response time of the small probing message to examine the condition of the transmission path between the mobile node and the server. In other words, if the mobile node receives the reply from the source quickly, it indicate that the quality of connection between the mobile node and the server is good enough. We also consider other two variations of ping-based methods - the ping-random2 (pr2) method and the random2ping ( $(\mathrm{r} 2 \mathrm{p})$ method. The ping-random 2 (pr2) method is similar to the ping-based method except that the mobile node has to choose one randomly from two servers that their ping response messages are most quickly returned. The random2-ping ( $\mathrm{r} 2 \mathrm{p}$ ) method is that the mobile node randomly selects two servers from the potential server list and then only sends out the ping messages to these two servers rather than all servers in the list. In $\mathrm{pr} 2$ and $\mathrm{r} 2 \mathrm{p}$ methods, the mobile node can not only select the proper server dynamically but free from connecting to the same server with other competing mobile nodes. Obviously, there is a number of other selection schemes that could be built or just reconstructed from the above ones. However, this set is reasonably representative for us to make comparisons.

\subsection{Popularity-Aware Channel Management Module}

In this section, we discuss some important issues in the channel management module including (i) when and how to create an ad hoc group, (ii) when and how to join an ex- 


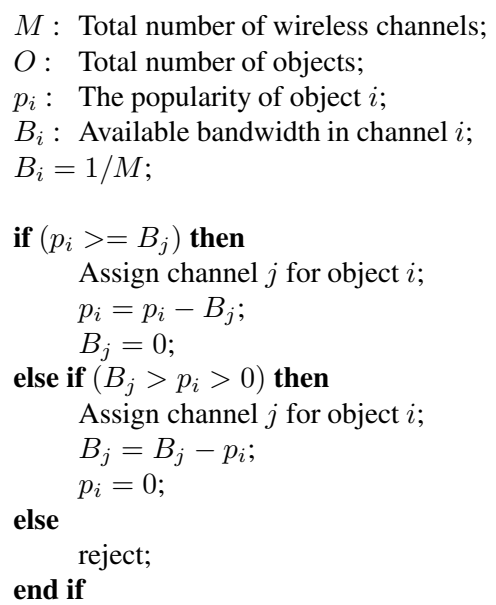

Figure 1. Channel management algorithm.

isting ad hoc group, and (iii) what should be done before a node leaves the ad hoc group.

3.2.1. Create an Ad Hoc Group: After the mobile node has got its requested objects and is willing to share those objects with other users, it can send the control message to the AP. After receiving this message, the AP determines if it is necessary to construct a new ad hoc group for those objects according to the channel management protocol. Since the popularity of accessed objects may be skew, an inappropriate channel assignment in the system may lead to poor resource utilization and system performance. We propose a popularity-aware channel management module and give its details as follows.

The main idea of the popularity-aware scheme is to allocate all available channels to each object in proportion to its popularity. A popular object should take advantage of more channels than a non-popular one to meet more access requests from mobile nodes. The detail about how to allocate channels is described in Figure 1. The bandwidth of all wireless channels is normalized to 1 , and will be fully utilized in our popularity-aware management protocol. Moreover, each object will get the suitable amount of bandwidth based on its popularity. When an AP receives the request from certain node, which wants to share object $i$ with the popularity $p_{i}$, it will check if $p_{i}$ is greater than 0 first. If so, the AP randomly chooses a channel, called channel $j$, which there is still free bandwidth in and allocate it to object $i$. The amount of bandwidth that could be allocated to this object is $\max \left(p_{i}, B_{j}\right)$ (the $\max (\mathrm{a}, \mathrm{b})$ function returns the larger one from two input values ), and $p_{i}$ and $B_{j}$ will both be reduced by $\max \left(p_{i}, B_{j}\right)$ after getting available bandwidth. After updating the value of $p_{i}$ and $B_{j}$, the object $i$ could not get any more resource if its popularity $p_{i}$ has been reduced to 0 , and the channel $j$ cannot be allocated to other objects if $B_{j}$ has been reduced to 0 .
3.2.2. Join an Ad Hoc Group: In H-WLAN framework, there are several conditions for a mobile node to join an existing ad hoc group. We describe them as follows:

1) Retrieving an object: The mobile node decides to get objects from another mobile node in some existing ad hoc group.

2) Sharing a wireless channel: The mobile node would like to share its objects but there are no more available wireless channels to build a new ad hoc group.

3) Out of range of APs: The mobile node cannot connect to any AP directly, i.e., there is no AP within its direct transmission range.

After deciding to join which ad hoc group, the mobile node has to send new state message including the group identification and the object list to the AP. The APs update their group information table and channel status table respectively after receiving this message.

3.2.3. Leave an Ad Hoc Group: After joining an ad hoc group, the mobile node might want to break the connection or get some objects that cannot be found in the current ad hoc group. Thus, the mobile node has to leave its ad hoc group to get the requested objects from other ad hoc groups or APs. In order to let the hybrid system work correctly, the mobile node should notify the AP to update all relevant information such as group information table and channel status table and take back the wireless channel before leaving. For instance, if some objects are only held on this leaving mobile node, the server has to delete this object field. If the group leader would like to leave, the APs have to appoint another group member as the new group leader before allowing it leaving or else this ad hoc group would be forced to be closed.

\section{Performance Evaluation}

We evaluate the performance of our H-WLAN framework by simulation, implemented in NS2 [4] with CMU wireless extension. In the simulation topology, there are 3 APs uniformly distributed in a $450 m \times 400 m$ area. The data rate of a wireless channel is $11 \mathrm{Mbps}$ and the transmission range of all mobile nodes, including the APs, is $250 \mathrm{~m}$. The total number of objects in the simulation is 10 . The object size is uniformly distributed between $100 \mathrm{~KB}$ and $1 \mathrm{MB}$. Another important observation is that users usually request the objects at some specific periods, such as the period before a meeting or a class, and hence the inter-arrival time of request is getting smaller while the scheduled time of a presentation (or a class) approaches. To consider the above observation, we use a 2-phase arrival process with 2 different arrival rates in the simulations. For this 2-phase arrival process, it has a lower mean arrival rate $\lambda_{l}$ for the first $k$ arrivals and a higher mean arrival rate $\lambda_{h}$ for the remaining arrivals. Moreover, let the random variables of $x_{l}$ and 
$x_{h}$ represent the interarrival times in the first phase and second phase and they are exponential distributions with means $\lambda_{l}=1$ and $\lambda_{h}=5(/ \mathrm{sec})$ accordingly.

The performance metrics used in the remainder of this section are: (a) the mean response time to get a requested object, (b) the variance of mean response time, (c) the ratio of control overhead, which is exchanged for keeping some information such as potential server list, to total bandwidth consumption, and (d) the makespan, the time needed to complete the transfer of all requested objects. These metrics will reflect the quality-of-service characteristics that would be of general interest to the wireless LAN system and users.

\subsection{Service Selection Study}

First, we investigate the performance of different wireless network systems under Poisson arrival case and then Bulk arrival case. Each AP uses a different wireless channel and in this simulations there are 2 additional wireless channels, which are available for setting up 2 new ad hoc groups to share the objects locally. We use the pure infrastructurebased WLAN as the baseline to be compared with the performance of H-WLAN.

4.1.1. Poisson arrival case: The request arrival pattern is the Poisson arrival. Each user needs 5 objects sequentially and the order of requested objects is randomly selected from all possible 10 objects. There are total 30 users and each of them expects to get 5 different objects. We evaluate the performance of different selection schemes respectively as follows:

1) Hybrid mod vs. Infrastructure mode: From Figure 2 and 3, the simulation results show that the hybrid system, i.e., random, ping, pr2, or $\mathrm{r} 2 \mathrm{p}$, outperforms the traditional infrastructure-based system, i.e., wlan, in terms of mean response time and makespan time. Such performance improvement for users is due to the fact that the hybrid system is able to make use of available wireless channels to increase both the system's capacity and the user's throughput. As the makespan time is concerned, the r2p method also has smaller mankspan than other methods; namely, using the ping-based methods, the mobile node can finish its schedule earlier. In addition to the wlan method, we consider an enhanced method for improving the performance of pure infrastructure mode, i.e., wlan-nc, which each AP keeps track of the number of connections and the AP with lowest load is assigned to next user's request. The comparison, illustrated in Figure 2 and 3, shows that our hybrid system, such as ping, pr2, and r2p, can still perform better than wlan-nc because of taking advantage of additional available wireless channels.

2) Ping-based vs. Random: Another important and interesting question arises which selection scheme is better and

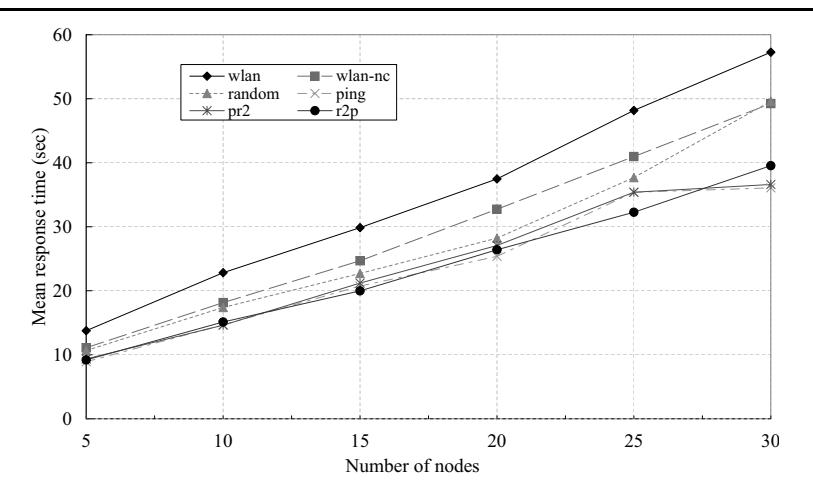

Figure 2. Poisson arrival: Mean response time.

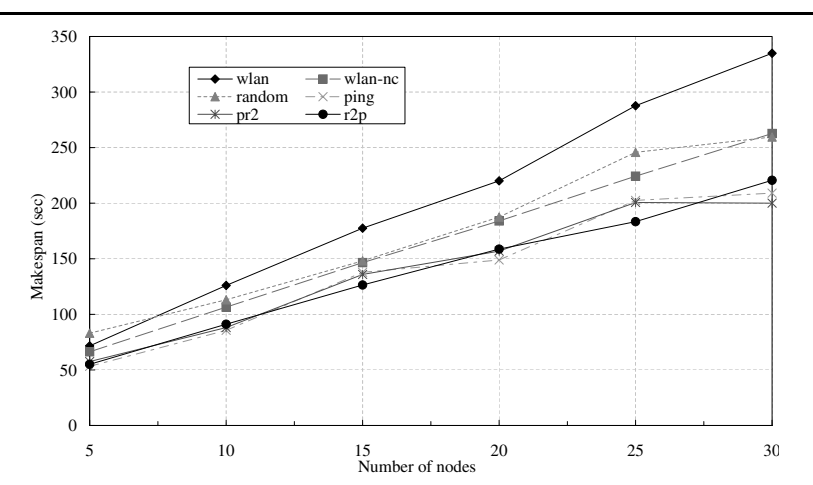

Figure 3. Poisson arrival: Makespan.

well-performing for the hybrid system. In Figure 2 and 3, we can see that the adaptive selection methods such as ping, pr2 and $\mathrm{r} 2 \mathrm{p}$ have smaller mean response time or makespan time than the random method. This can be explained as follows. In all adaptive selection methods, the mobile node can choose a better server and channel to use according to the network condition at that moment; nevertheless, in the random method, it just blindly chooses one of feasible servers to connect. In other words, in the random method, it is more likely to choose the worst (highest-loaded) or farthest server to connect. In our simulations, we do not consider the scenario with other background traffic, but, however, in a more practical network environment, it is not easy for the random method to achieve the load balance if there exist different amount of background traffic running on the servers. On the contrary, the mobile nodes, in the ping-based methods, can choose the suitable server dynamically via the information from ping response messages. Therefore, we will focus our discussion on ping-based methods, i.e., ping, pr2, and $\mathrm{r} 2 \mathrm{p}$, and tell the differences among them in the following sections.

3) Control Overhead Comparison: In this experiment, we assume that the infrastructure-based WLAN, i.e., the wlan method, does not require additional wireless band- 


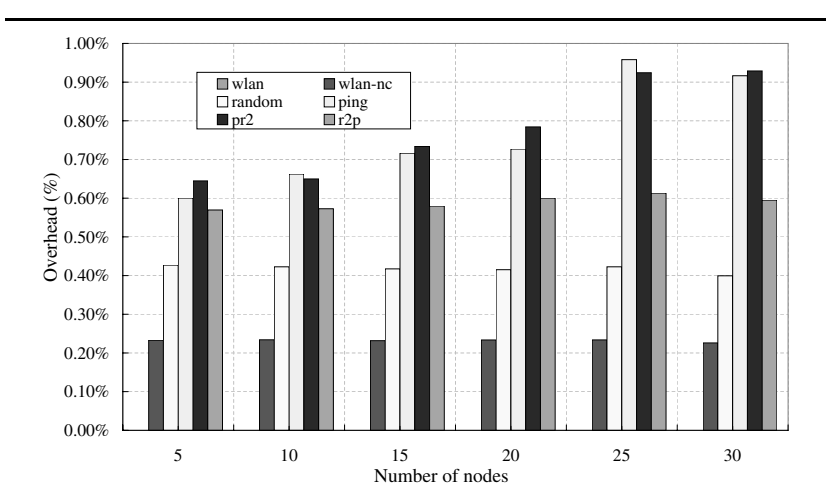

Figure 4. Poisson arrival: Overhead.

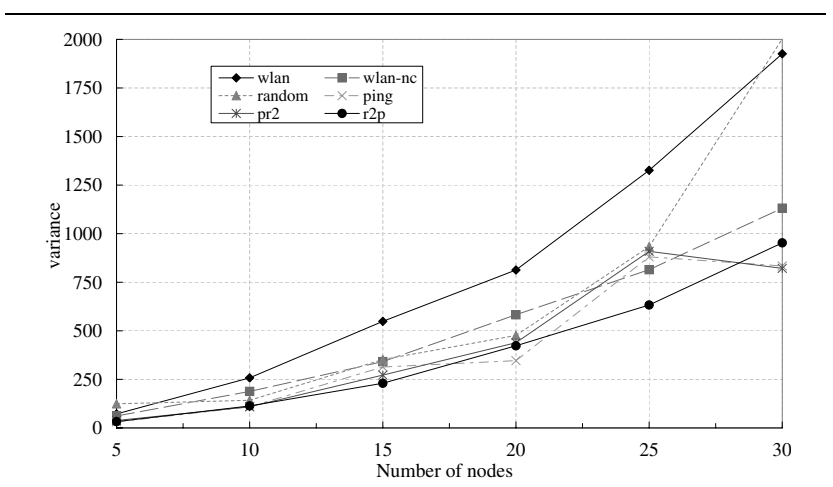

Figure 5. Poisson arrival: Variance.

width for exchanging control messages. In contrast, both the wlan-nc method and the hybrid methods do require additional wireless bandwidth to exchange information like the server list. The bandwidth requirements are normalized by all transferred data packets including the requested objects and the control messages. As the results shown in Figure 4, the bandwidth overhead of $r 2 p$ is no more than $0.6 \%$ while the ping and pr2 methods result in the bandwidth overheads that don't exceed $1 \%$. It shows that our H-WLAN can have a substantial performance improvement but the additional bandwidth requirement is small relatively.

4) Variance Comparison: As illustrated in Figure 5, the random method causes the higher variance compared with the rest of selection methods. This is not surprising since the random method is to select a server randomly from the server list without any further information; namely, the probability is equal for a mobile node to choose the nearest server or the farthest one. The rest of adaptive hybrid methods such as ping, pr2, and $\mathrm{r} 2 \mathrm{p}$ can provide fair and consistent service (mean response time) compared with the random method under this simulation setup.

4.1.2. Bulk arrival case: After the above discussions, an important and interesting question arises: which service selection method is most suitable for the hybrid system. Thus,

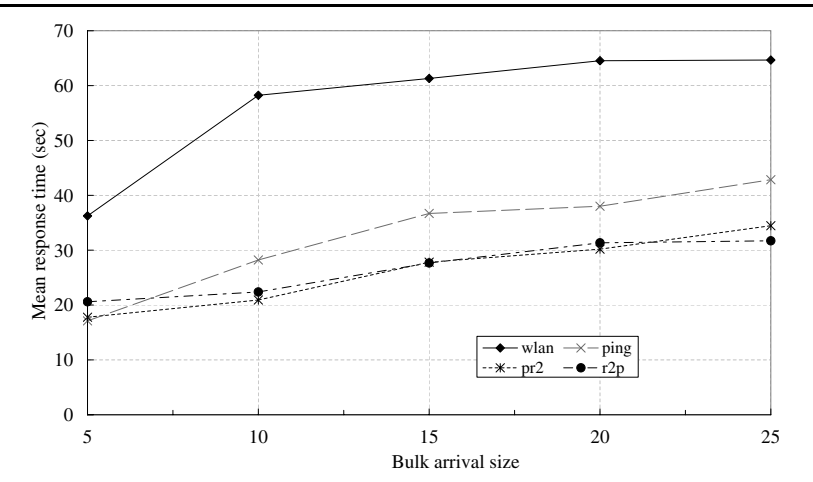

Figure 6. Bulk arrival: Mean response time.

we consider a more bursty arrival pattern and observe the performances under the bursty network condition. In this simulation, all mobile nodes want to obtain the same single object but the number of each arriving nodes may be greater than 1 . That is, the bulk arrival size of first 5 arrivals is 1 and the bulk arrival size of the remaining arrivals is $s b$, where $s b$ is from 5 to 25 . There are total 30 mobile users who are uniformly distributed within the simulation area.

As shown in Figure 6, both pr2 and r2p methods can maintain lower mean response time compared with the ping method, where we can make the following observations. As the arrival is not bursty, i.e., the bulk arrival size is small, the ping method can make a good decision to choose a suitable server. However, when the bulk arrival size increases, it is not a good idea for all the users to choose its best server since they might choose the same one and swamp it. Consequently, the mobile node can avoid selecting the worst server and at the same time keep a load balance among all available servers. This is why the pr 2 and $r 2 p$ perform better than the ping method does.

\subsection{Channel Management Study}

Since $\mathrm{r} 2 \mathrm{p}$ method performs better than or as well as other server-channel selection schemes and only needs small control overhead, we use it to evaluate the performance of popularity-aware channel management module. In this experiment, the popularity of objects is the Zipf distribution, given in Eq. (1), and we set $\theta=0$ and $K=10$.

$$
\begin{aligned}
& \text { Prob[requent of object } i]=\frac{c}{i^{(1-\theta)}} \\
& \forall i=1,2, \ldots, K \text { and } 0 \leq \theta \leq 1 \\
& \text { Where } c=\frac{1}{H_{K}^{(1-\theta)}} \text { and } H_{K}^{(1-\theta)}=\sum_{j=1}^{K} \frac{1}{j^{(1-\theta)}}
\end{aligned}
$$

Each mobile node desires to get a single object, which is randomly selected from 10 possible objects. There are 5 additional wireless channels could be allocated for all objects to construct the ad hoc group. We use the first- $k$ and round- 


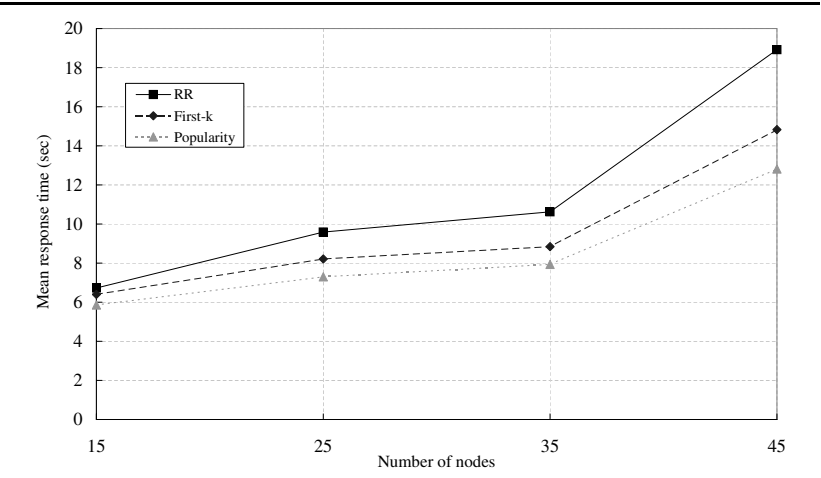

Figure 7. Channel management.

robin (RR) method as the base case for comparing with our popularity-aware mechanism.

In first- $k$ method, all available channels can only be allocated to first $k$ request objects, and each object may get multiple channels if it requests more than once and the request is still first- $k$ ones. In this simulation setup, if one channel can be only allocated to single object, 5 wireless channels can only respond to first 5 requests. When the popularity of object is uniform distribution, first- $k$ method might perform worse owing to the low utilization of bandwidth, i.e., the channels are not fully utilized but also not able to be allocated to other objects. On the other hand, it gets the acceptable performance, as shown in Figure 7, when the popularity of object is non-uniform, since the first 5 requests, which get the wireless channels to set up new ad hoc groups, may happen to share the more popular object and use bandwidth adequately.

In the RR method, the APs allocate the channel by round-robin whether all bandwidth in this channel is fully used or not, and authorize objects to get the resource by round-robin as well, i.e., if an object was once authorized to get the channel, it is able to get additional channel unless all other objects have been allowed to get the channel once. It is fair for every objects but not a good scheme for this case that the popularity of objects is the Zipf distribution. As illustrated in Figure 7, the round-robin method has the worst performance, compared to others, due to improper management. The more popular objects can not get sufficient bandwidth to use, but at the same time the bandwidth that is allocated to non-popular objects is still idle. Moreover, the results show that our popularity-aware mechanism has the best performance under the skew popularity. The lower mean response time also illustrates that the users can get a better QoS and the total amount of bandwidth can be utilized more effectively.

\section{Conclusions}

In this paper, we discussed the issues about the integration of ad hoc mode wireless network with infrastructurebased WLAN for the data dissemination applications. The main contributions of H-WLAN are as follows. First, we have shown that it is promising to use extra available channels to set up the ad hoc groups for aiding the traditional infrastructure-based WLAN, and the simulation results also illustrate that the QoS of users and the throughput and utilization of system are both substantially enhanced in $\mathrm{H}$ WLAN. Second, the adaptive service selection module and the popularity-aware channel management module are proposed in this work. Both modules can provide the efficient resource management and consistent service. The mobility issue of nodes is beyond the focus of this work since the mobility may lead to separation in an ad hoc group and termination in current connections. Our future work plans to take the mobility issue into consideration.

\section{References}

[1] Y. Bejerano. Effi cient integration of multi-hop wireless and wired networks with qos constraints. In Proceedings of ACM Mobicom, Sep. 2002.

[2] J. Chen, S. G. Chan, J. He, and S. Liew. Mixed-mode wlan: The integration of ad hoc mode with wireless lan infrastructure. In Proceedings of IEEE Globecom, Dec. 2003.

[3] W. C. Cheng, C.-F. Chou, L. Golubchik, and S. Khuller. A performance study of bistro, a scalable upload architecture. In In ACM SIGMETRICS Performance Evaluation Review, pages 31-39, March 2002.

[4] http://www.isi.edu/nsnam/ns/. The Network Simulator - ns2.

[5] S. B. Kodeswaran, O. Ratsimor, A. Joshi, T. Finin, and Y. Yesha. Using peer-to-peer data routing for infrastructurebased wireless networks. In PerCom, page 109120, Mar. 2003.

[6] Y. Sun and E. M. Belding-Royer. Application-oriented routing in hybrid wireless networks. In Proceedings of ACM Mobicom, May 2003.

[7] P. U. WICAT. Information Project. http://wicat.poly.edu/infostation.htm.

[8] T. Ye, H.-A. Jacobsen, and R. H. Katz. Mobile awareness in a wide area wireless network of info-stations. In Mobile Computing and Networking, pages 109-120, 1998.

[9] L. Yin and G. Cao. Supporting cooperative caching in ad hoc networks. In Proceedings of IEEE INFOCOM, Mar. 2004.

[10] W. H. Yuen, R. D. Yates, and S.-C. Mau. Noncooperative content distribution in mobile infostation networks. In IEEE WCNC, Mar. 2003. 